\title{
Exploring the Effects of Creating Small High Schools on Daily Attendance: A Statistical Case Study
}

\author{
MATTHIJS KOOPMANS \\ Mercy College (USA)
}

Does creating small high schools have a beneficial impact on daily attendance? This question was addressed using time series analysis to examine the case of one urban transfer high school that serves students who previously dropped out of school. This analytical approach is uniquely suitable to examine the dynamical processes characterizing stability and transformation in the system. This school reduced its size from enrolling approximately 900 students up to and through the 2009-2010 school year to about 250 students afterward. We looked at whether attendance was higher after the intervention and whether it was more stable. It turns out that the attendance trajectories over a seven-year period show high volatility prior to the reduction in school size but are more stable afterward. The initial increase in daily attendance at the onset of the intervention is not maintained, but increases are observed later. The study illustrates the relevance of time series analysis for educational policy research as well as the use of complexity theory to fully appreciate the nature of the post intervention changes.

School attendance is a complex phenomenon. It is a behavioral expression of the interplay of a causal network that includes such critical variables as parental support, socio-economic status of students' families, instructional quality and resources at the school serving the students, a network of variables between schools and families regarding education and teacher supportiveness toward individual students. School attendance is also complex in the sense that it may be highly irregular over time. While we know that attendance is a critical factor to academic success, its determinants are under-researched. The research reported here is part of a program of ongoing study of the long range patterns of daily attendance in New York City high schools, and the identification of signs of complex adaptive behavior (fractality, meta-stability) in those patterns. Previous findings were reported by Koopmans (2015a; 2015b; 2016; 2017a; 2017b; 2018).

Fractality, or self-similarity, refers to patterns replicating within themselves, a feature that has been interpreted in the dynamical literature as an indicator of a complex adaptive process while a system retains its characteristics under changing circumstances (Beran, 1994; Mandelbrot, 1997; Stadnitski, 2012). Meta-stability refers to a state where repeated exposure to the same input conditions creates heightened tension in the system because of which change is imminent. Hence, it is also referred to as self-organized criticality or the edge of chaos (Bak, 1996; Jensen, 1998). Fractality and meta-stability are of interest because they bring an element of uncertainty into our observation and interpretation of attendance patterns in school buildings.

School practitioners will be able to predict regularly occurring cycles of variation such as those coinciding with the days of the week, month and school year. Irregular patterns are harder to

Complicity: An International Journal of Complexity and Education

Volume 15 (2018), Number $1 \bullet p p .19-30$ 
detect, but they may be important predictors of attendance behavior and therefore, studying them is of interest.

Koopmans (2018) compares daily attendance rates in small vs. large high schools, and finds that, although random variability is greater in the small schools, fractal and meta-stable patterns are found to the same degree in both types of schools. The present study addresses the relationship between school size and dynamical characteristics of school attendance trajectories from a different angle by studying a school whose size was drastically reduced, thereby creating an opportunity for a pre-post intervention comparison of the attendance rates, their variability and underlying dynamics.

The question whether creating smaller high schools has a favorable impact on school success is especially relevant when schools serve vulnerable populations, such as those with lower prospects of graduating. The school used for this study, referred to here as the Circle Academy, defines as its mission to 'help students overcome their negative feelings about school, improve their attendance, become active learners and graduate with full capability for future success.' (New York City Department of Education, 2017). This school underwent a drastic reduction in size starting in the fall of 2010 to create an environment conducive to those aims. Are attendance rates higher in this school after than before this transition? Do they show greater stability and predictability afterward? These questions cannot be fully addressed through conventional summary statistics (mean, range), as they require a detailed description of the sequential order of the attendance rates over a longer period. The methodology to analyze such information is available (e.g., Beran, 1994), but is used infrequently in education. The analysis presented here focuses on meta-stability. The occurrence of self-similarity is not further pursued here, but see Koopmans $(2016 ; 2018)$.

\section{Method}

In this study, the daily attendance rates in one public school in New York City, the Circle Academy, are analyzed over a seven-year period, i.e., from September 2007 through June 2014 ( $N$ $=1,245)$. The school enrolled approximately 900 students up to and through the 2009-2010 school year. Afterward, enrollment had been reduced to about 250 students and remained stable for the period under study. A continuous trajectory was created by removing the vacations, holidays, intersessions and weeks that had three days or more missing. If fewer than three days were missing from any given week, the median of the original series was imputed.

The New York City's Department of Education's publicly available data permit a determination of enrollment as well as the demographic composition of the student body in the period of interest (see Table 1). A pre-intervention (2007-2010) and post-intervention period (2010-2014) was distinguished in subsequent analyses based on this information, as shown in Table 2 further below.

Complicity: An International Journal of Complexity and Education

Volume 15 (2018), Number $1 \bullet p p .19-30$ 
Table 1. Total Enrollment and Percent of Students in Each Demographic Category per School Year at the Circle Academy

\begin{tabular}{ccccccc}
\hline & Enrollment & Female & Non-white & $\begin{array}{c}\text { Students } \\
\text { with } \\
\text { Disabilities }\end{array}$ & $\begin{array}{c}\text { English } \\
\text { Language } \\
\text { Learners }\end{array}$ & $\begin{array}{c}\text { Free or } \\
\text { Reduced- } \\
\text { Price Lunch } \\
\text { Eligibility }\end{array}$ \\
\cline { 2 - 6 } $\begin{array}{c}\text { School Year } \\
2007-08\end{array}$ & 959 & $52.8 \%$ & $96.4 \%$ & $9.3 \%$ & $2.7 \%$ & $68.5 \%$ \\
$2008-09$ & 901 & $54.8 \%$ & $96.8 \%$ & $9.1 \%$ & $2.4 \%$ & $73.6 \%$ \\
$2009-10$ & 910 & $55.4 \%$ & $97.2 \%$ & $8.5 \%$ & $2.5 \%$ & $76.6 \%$ \\
$2010-11$ & 274 & $57.7 \%$ & $96.7 \%$ & $12.0 \%$ & $2.6 \%$ & $79.9 \%$ \\
$2011-12$ & 248 & $61.3 \%$ & $97.1 \%$ & $14.1 \%$ & $6.5 \%$ & $84.3 \%$ \\
$2012-13$ & 247 & $60.3 \%$ & $97.9 \%$ & $16.2 \%$ & $6.1 \%$ & $84.6 \%$ \\
$2013-14$ & 255 & $54.9 \%$ & $98.0 \%$ & $14.5 \%$ & $3.5 \%$ & $87.5 \%$ \\
\hline
\end{tabular}

Three complementary analytical strategies were executed to estimate the effects of the enrollment reduction at the Circle Academy. First, the autoregressive and moving average components to the variability in the time series were estimated, using a conventional Autoregressive Integrated Moving Average (ARIMA) framework (Cryer \& Chan, 2008). Assuming a time series trajectory $Y_{t}, Y_{t-1}, Y_{t-2}, \ldots, Y_{t-n}$, autoregression can be estimated at $p$ lags, i.e., $\operatorname{AR}(p)$, as follows:

$$
Y_{t}=\phi_{1} Y_{t-1}+\phi_{2} Y_{t-2}+\cdots+\phi_{p} Y_{t-p}
$$

A general moving average model estimates the disturbances $e_{t}$ in $Y_{t}$ at $q$ lags, $\operatorname{MA}(q)$ as shown below:

$$
Y_{t}=\mu+e_{t}-\theta_{1} e_{t-1}-\theta_{2} e_{t-2}-\cdots-\theta_{q} e_{t-q}
$$

The present analysis focuses on the estimation of seasonal dependencies in these two processes at five lags, i.e., SARIMA $(1,1)_{5}$, representing the days of the school week.

Second, the statistical properties of the temporal process before and after the transition were examined for signs of meta-stability and self-organized criticality (Bak, 1996), using a fractional differencing approach (Beran, 1994). The recognition of meta-stability relies on the estimation of a differencing parameter $d$, which depicts the extent of long range dependency between observations across the entire series, or it's 'stickiness', also referred to in the literature as pink noise, $1 / f$ noise or persistence (Stadnitski, 2012). The fractional difference is typically defined in terms of a backshift operator $B$, i.e., $B\left(Y_{t}\right)=Y_{t-1}, B^{2}\left(Y_{t-1}\right)=Y_{t-2}, \ldots, B^{n}\left(Y_{t-n-1}\right)=Y_{t-n}$, where the higher order terms represent the diminishing influence of preceding observations as lag size increases (e.g., Cryer \& Chan, 2008). Fractional differencing estimates the long range dependency as follows:

$$
(1-B)^{\mathrm{d}} Y_{t}=e_{t}
$$


assuming $-.5<d<.5$, such that $d=0$ (no long-term autocorrelation), $d>0$ (positive long term autocorrelation or persistence) or $d<0$ (negative long term autocorrelation, or anti-persistence, Beran, 1994; Sowell, 1992).

In a stationary series, i.e., a series with constant statistical properties across the time scale, $Y_{t}$ normally gets analyzed. In a volatile series, the first difference $Y_{t}-Y_{t-1}$ is typically taken, or a multiple thereof, to attain stationarity before proceeding with the analysis. In the former case, $d=0$, in which case the term depicted in (3) disappears; in the latter case, $\mathrm{d}=1,2, \ldots$, an integer represents the lag at which the difference is taken to remove non-stationarity. Pink or $1 / f$ noise represents the intermediate case between stationarity and non-stationarity in that equation (3) provides a statistical correction to address the non-random variability not captured by the traditional time series estimates shown in equations (1) and (2) above. In the dynamical literature a fractional difference is said to be suggestive of meta-stability or self-organized criticality in the system of interest (Bak, 1996; Mandelbrot, 1997).

Third, the impact of perturbation on the series was estimated, particularly at the point of transition from a large to a small school, using intervention analysis, or pulse modelling (Peña, 2001). Pulse modeling incorporates the effects of outlying values on the series into the analysis, utilizing the following general model:

$$
e_{t}=\omega Y_{t}+a_{t}
$$

which shows the relationship between the residual $e_{t}$ and the unperturbed model $Y_{t}$ as a regression model with the following test statistic:

$$
\lambda_{j}^{T}=\omega_{j}^{T} /\left(\sqrt{\operatorname{Var}\left(\omega_{j}^{T}\right)} \quad \sim N(0,1)\right.
$$

to decide if an observation qualifies as an outlier. The pulse analyses investigated three scenarios to mark a divergence of individual data points from the entire series:

- Additive Outliers, where the value $\omega$ represents the size of a one-time departure from the unperturbed series at a given time point, with a parameter estimate marking the size of their departure from the central tendency of the series,

- Level Shift Outliers, where $\omega$ marks a 'permanent' departure from the unperturbed series from a given time point onward, and

- Transient Change Outliers, in which $\omega$ indicates a change at a given point followed by an estimated recovery rate $(\delta)$ toward the central tendency of the unperturbed series. A full mathematical explication of these scenarios is provided by Koopmans (2017b) and Peña (2001).

The three analyses described above handled different aspects of the evaluation of the impact of the school size change on attendance behavior. For each analysis, it was crucial to make the distinction between the pre-intervention and post-intervention period at $t=546$, or September 13 , 2010. For the pulse models, the entire series was analyzed and the impact of all outliers was estimated, including those associated with the shift to post-intervention. For the factional 
differencing, the pre-intervention and post-intervention period were defined as separate time series to investigate their stability, meta-stability or volatility. This analysis included an initial assessment about whether the statistical properties of each of the series were constant (stationarity), and if so, the extent to which they showed persistence. The Augmented DickeyFuller (ADF) test (Said \& Dickey, 1984) was used to decide on the stationarity of the data as part of the initial data exploration, and its results are shown in Table 2.

Table 2. Daily Attendance Summary Statistics and Stationarity Test Results: Pre- and PostIntervention

\begin{tabular}{lcccccccc} 
& Lowest & $\mathbf{1}^{\text {st }} \mathbf{Q}$ & Median & $3^{\text {rd }} \mathbf{Q}$ & Highest & Mean & $\mathbf{N}$ & ADF \\
\cline { 2 - 8 } & & & & & & & & \\
Status & & & & & & & & \\
Before $(\mathrm{N}=545)$ & 41.89 & 65.97 & 69.15 & 72.73 & 83.20 & 68.78 & 545 & -3.15 \\
After $(\mathrm{N}=700)$ & 27.83 & 70.74 & 77.94 & 81.73 & 94.76 & 75.53 & 700 & $-4.57^{*}$ \\
Total Series & 27.83 & 67.54 & 72.75 & 78.92 & 94.76 & 72.57 & 1,245 & $-4.81^{*}$ \\
\hline
\end{tabular}

${ }^{*} \mathrm{p}<.01$. A rejection of the null hypothesis implies stationarity. Lag order $=8$ before and after; Lag order $=10$ for the entire series.

Autocorrelation function plots were generated for an initial exploration of the dependencies in the data, and a competitive model selection procedure (Wagenmakers, Farell, \& Ratcliff, 2004) was followed to estimate the relative importance of short range dependencies, weakly seasonal dependencies (five lags for the days of the school week) and the long range dependencies or persistence (post-intervention data only). The Bayesian Information Criterion (BIC) was used to select the best fitting pre and post models, and parameter estimates of those models were further interpreted.

\section{Results}

Figure 1 shows the attendance rates and the associated autocorrelation function plots (ACF) at 100 lags. The two left panels show the pre-intervention data and the panels in the middle show the post-intervention data. The panels on the right show the entire series. Comparing the trajectories pre and post leaves the impression of greater volatility in the pre-intervention trajectory, but also continuing variability afterward. The spikes in the post-intervention ACF plots indicate seasonal cycles that appear to be more pronounced than in the pre-intervention plot. Tables $3 \mathrm{a}$ and $3 \mathrm{~b}$ summarize the results of the pulse analysis for the pre and post-intervention trajectory, respectively. The estimates are based on a single model for the entire trajectory. The onset of the small school arrangement creates a pulse on Sept. 13 and Sept. 14, 2010 ( $t=546$ and 547 , marked in red) of the transient kind, which is to say that from there, a 'recovery' is seen to the central tendency of the original series. However, there are also positive level shifts in the postintervention period at the beginning of the school year in $2012(t=896)$ and toward the end of the school year in $2014(t=1,240)$. 

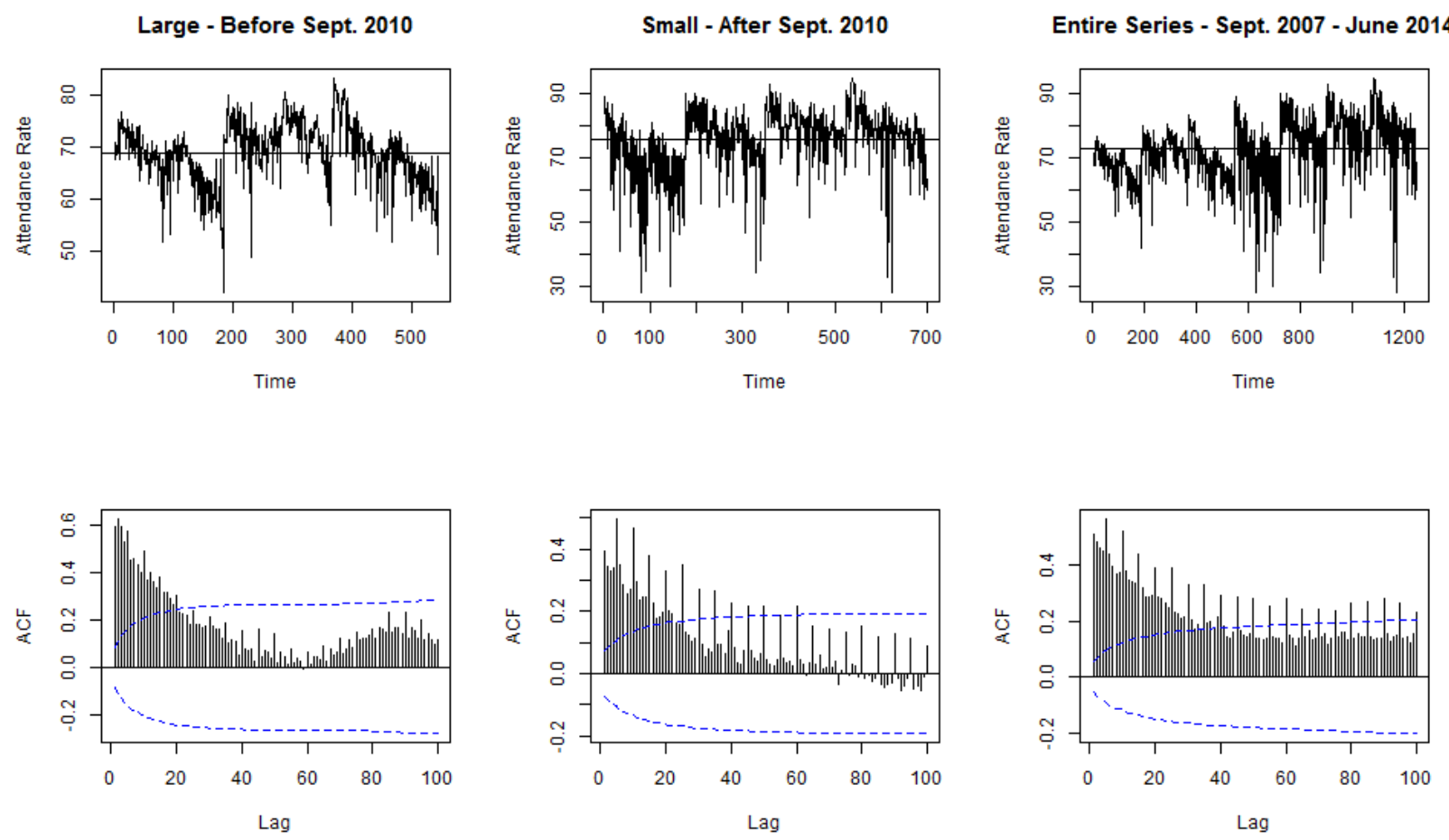

Figure 1. Attendance Rates and Autocorrelation Function (ACF) Plots Before and After the Circle Academy Became a Small High School. Left: Pre-intervention, Middle: Post-intervention, Right: Entire Period. Straight lines in the attendance plots represent the mean of the series. Dotted lines in the ACF plots represent the $95 \%$ confidence interval.

Table 3a. Outlier Impact Estimates by Outlier Type: Pre-Intervention

\begin{tabular}{lcccc}
\hline Outlier Type & $\begin{array}{c}\text { Pulse } \\
\boldsymbol{\omega}(\mathbf{s e})\end{array}$ & $\begin{array}{c}\text { Recovery } \\
\boldsymbol{\delta}(\mathbf{s e})\end{array}$ & Location $\mathbf{t}=\mathbf{~ T}$ & Date \\
\cline { 2 - 5 } Transient Change & $-7.71(1.07)$ & $.99(.00)$ & 150 & $4 / 18 / 2008$ \\
(TC) & $13.89(1.49)$ & $.99(.00)$ & 191 & $9 / 8 / 2008$ \\
& $10.49(1.79)$ & $.98(.01)$ & 284 & $2 / 5 / 2009$ \\
& $14.27(2.07)$ & $.96(.01)$ & 371 & $9 / 14 / 2009$ \\
& $-6.57(1.19)$ & $1.00(.00)$ & 515 & $4 / 30 / 2010$ \\
Additive (AO) & & & & \\
& $-19.50(5.29)$ & -- & 150 & $4 / 18 / 2008$ \\
& $-22.92(5.28)$ & -- & 230 & $10 / 31 / 2008$ \\
\hline
\end{tabular}

The ADF test results shown in Table 2 indicate that while the post-intervention attendance trajectory is stationary, the pre-intervention trajectory is not. Therefore, the first difference $Y_{t}-Y_{t}$ ${ }_{1}$ i.e., $d=1$, was taken to examine the short range and seasonal error dependencies in the time 
increments of the series, as is common practice in these types of analyses (e.g., Cryer \& Chan, 2008).

Table 3b. Outlier Impact Parameter Estimates by Outlier Type: Post-Intervention

\begin{tabular}{|c|c|c|c|c|}
\hline Outlier Type & $\begin{array}{l}\text { Pulse } \\
\omega(\text { (se) }\end{array}$ & $\begin{array}{c}\text { Recovery } \\
\delta \text { (se) }\end{array}$ & Location $t=T$ & Date \\
\hline Transient Change & $16.43(5.08)$ & $.98(.10)$ & 546 & $9 / 13 / 2010$ \\
\hline \multirow[t]{3}{*}{ (TC) } & $7.62(5.57)$ & $.99(.00)$ & 547 & $9 / 14 / 2010$ \\
\hline & $20.85(1.23)$ & $.99(.00)$ & 721 & $9 / 12 / 2011$ \\
\hline & $14.05(1.73)$ & $.97(.01)$ & 1,081 & $9 / 23 / 2013$ \\
\hline \multirow{2}{*}{ Level Shift (LS) } & $11.44(0.99)$ & -- & 896 & $9 / 10 / 2012$ \\
\hline & $11.80(2.26)$ & -- & 1,240 & $6 / 6 / 2014$ \\
\hline \multirow[t]{21}{*}{ Additive (AO) } & $-23.39(5.28)$ & -- & 568 & $10 / 13 / 2010$ \\
\hline & $-33.24(5.28)$ & -- & 580 & $10 / 29 / 2010$ \\
\hline & $-22.08(5.27)$ & -- & 605 & $12 / 03 / 2010$ \\
\hline & $-29.02(5.25)$ & -- & 625 & $1 / 7 / 2011$ \\
\hline & $-40.02(5.25)$ & -- & 628 & $1 / 12 / 2011$ \\
\hline & $-21.50(5.25)$ & -- & 630 & $1 / 14 / 2011$ \\
\hline & $-24.58(5.25)$ & -- & 635 & $1 / 21 / 2011$ \\
\hline & $-32.78(5.25)$ & -- & 638 & $2 / 2 / 2011$ \\
\hline & $-25.38(5.25)$ & -- & 665 & $3 / 18 / 2011$ \\
\hline & $-35.40(5.26)$ & -- & 690 & $4 / 29 / 2011$ \\
\hline & $-26.22(5.26)$ & -- & 755 & $10 / 28 / 2011$ \\
\hline & $-23.71(5.24)$ & -- & 795 & $12 / 23 / 2011$ \\
\hline & $-27.57(5.25)$ & -- & 850 & $3 / 30 / 2012$ \\
\hline & $-38.85(5.26)$ & -- & 875 & $5 / 11 / 2012$ \\
\hline & $-34.31(5.26)$ & -- & 885 & $5 / 25 / 2012$ \\
\hline & $-22.59(5.27)$ & -- & 890 & $6 / 1 / 2012$ \\
\hline & $-27.86(5.24)$ & -- & 990 & $2 / 8 / 2013$ \\
\hline & $-24.39(5.26)$ & -- & 1,147 & $1 / 7 / 2014$ \\
\hline & $-44.61(5.25)$ & -- & 1,158 & $1 / 22 / 2014$ \\
\hline & $-33.23(5.25)$ & -- & 1,163 & $2 / 5 / 2014$ \\
\hline & $-48.96(5.25)$ & -- & 1,169 & $2 / 13 / 2014$ \\
\hline
\end{tabular}

Fractional differencing models were fitted to the original post-intervention trajectories to estimate short range, seasonal and long range dependencies. Table 4 summarizes the model selection process for both data segments, including the autoregressive (AR), moving average (MA) components (with $S$ as a seasonal prefix at five lags) and differencing parameter $(d)$. The best fitting pre-intervention model (Model 3) includes seasonal variance components at five lags (days of the week). The best-fitting post-intervention model (Model 6) also includes a statistically significant long-range effect. Figures 2 and 3 show the series that were analyzed in the pre and post-intervention case, respectively, as well as the ACF diagnostics for some of the models fitted.

Complicity: An International Journal of Complexity and Education Volume 15 (2018), Number 1・pp. 19-30 
Table 4. Model Selection and Goodness of Fit Pre and Post-Intervention: Log Likelihood Estimates and Bayes Information Criterion (BIC)

\begin{tabular}{|c|c|c|c|}
\hline Model & Specification & LL & BIC \\
\hline \multicolumn{4}{|c|}{ Pre-Intervention } \\
\hline 1 & $\mathrm{~d}=1$ & $-1,994.54$ & $3,995.34$ \\
\hline 2 & $\operatorname{MA}(12), d=1$ & $-1,568.79$ & $3,150.18$ \\
\hline 3 * & $\operatorname{MA}(12), \operatorname{SAR}_{5}(1), \operatorname{SMA}_{5}(1), \mathrm{d}=1$ & $-1,532.28$ & $3,089.76$ \\
\hline \multicolumn{4}{|c|}{ Post-Intervention } \\
\hline 1 & Int. & $-2,608.99$ & $5,224.53$ \\
\hline 2 & Int., $\mathrm{d}$ & $-2,503.01$ & $5,019.12$ \\
\hline 3 & Int., $\mathrm{AR}(1), \mathrm{MA}(1)$ & $-2,486.97$ & $4,993.59$ \\
\hline 4 & Int. , $\mathrm{AR}(1), \mathrm{MA}(1) \mathrm{XSAR}_{5}(1)$ & $-2,464.46$ & $4,955.12$ \\
\hline 5 * & Int., $\mathrm{AR}(1), \mathrm{MA}(1) \mathrm{XSAR}_{5}(1258$ 12) & $-2,416.54$ & $4,885.49$ \\
\hline 6 * & Int., $\mathrm{AR}(15), \mathrm{MA}(15), \mathrm{d}$ & $-2,419.79$ & $4,878.89$ \\
\hline
\end{tabular}

* Preferred models
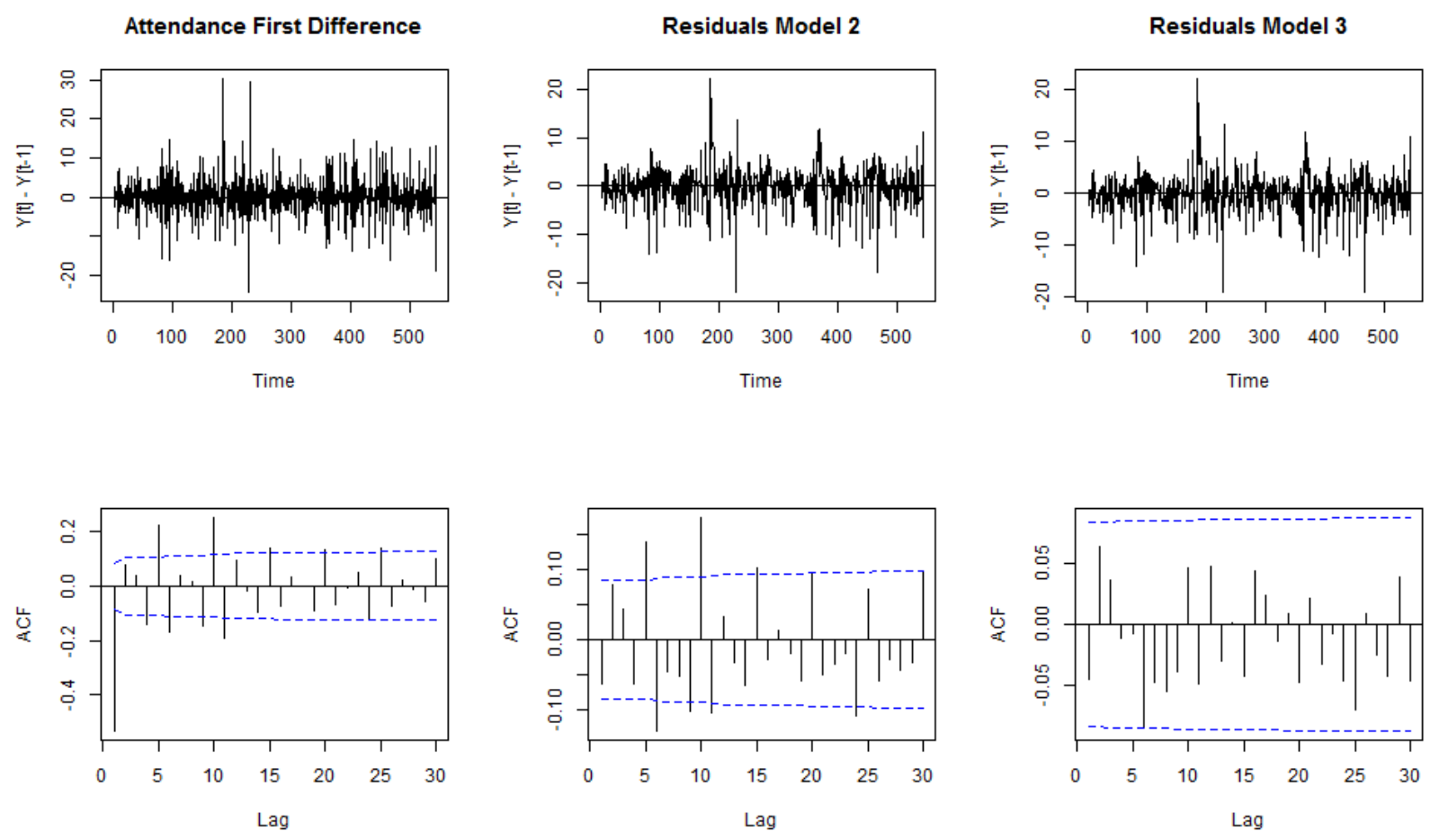

Complicity: An International Journal of Complexity and Education Volume 15 (2018), Number $1 \bullet p p .19$ - 30 
Figure 2. Attendance Rates (First Difference) and Autocorrelation Plots (30 Lags) of the PreIntervention Series. Left: First Difference $\left(Y_{t}-Y_{t-1}\right)$ of the Original Series, Middle: Residuals Model 2, Right: Residuals Model 3.

The residuals for Model 2 shown in Figure 2 clearly show the remaining seasonal dependencies as spikes in the ACF plot at the fifth lag, whereas the residuals for Model 3 show their successful removal by the seasonal model. Figure 3 shows that both Model 5 and Model 6 are effective in that remaining autocorrelations in the ACF plot show random patterns that are largely not statistically significant. The parsimony of the data description in Model 6 yields a better goodness of fit. The coefficients in Table 5 shows the statistical significance of the seasonal components in both the pre and post-intervention models, as well as the significance of the longrange dependencies in the post-intervention model.
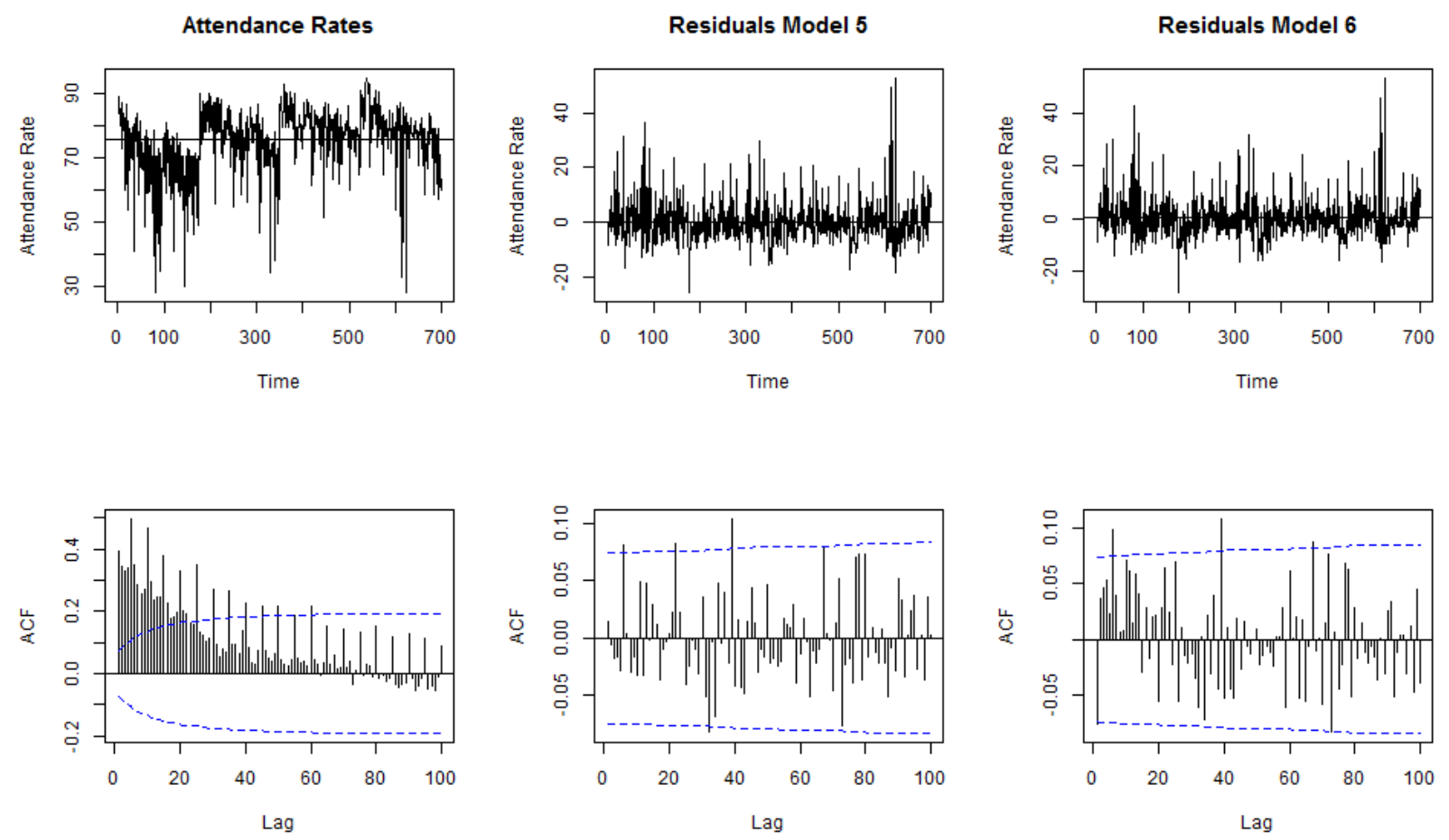

Figure 3. Attendance Rates and ACF Plots of the Post-Intervention Series: Left: Original Series, Middle: Residuals Model 5, Right: Residuals Model 6 
Table 5. Parameter Estimates and Standard Errors of Best-Fitting Pre and Post-Intervention Models

\begin{tabular}{lcc}
\hline Coefficient & $\begin{array}{c}\text { Pre-intervention } \\
\text { Model 3 }\end{array}$ & $\begin{array}{c}\text { Post-Intervention } \\
\text { Model 6 }\end{array}$ \\
\cline { 2 - 3 } Intercept & -- & 75.15 \\
$(\mathrm{se})$ & & $(8.17)$ \\
$\varphi_{1}$ & --.01 \\
$(\mathrm{se})$ & & $(.01)$ \\
$\varphi_{5}$ & -- & .99 \\
$(\mathrm{se})$ & -1.67 & $(.01)$ \\
$\theta_{1}$ & .05 \\
$(\mathrm{se})$ & $(.03)$ & $(.02)$ \\
$\theta_{2}$ & .67 & -- \\
$(\mathrm{se})$ & $(.03)$ & -.91 \\
$\theta_{5}$ & & $(.03)$ \\
$(\mathrm{se})$ & -- & -- \\
$\Phi_{1(5)}$ & \\
$(\mathrm{se})$ & .99 & -- \\
$\Theta_{1(5)}$ & $(.00)$ & .26 \\
$(\mathrm{se})$ & -.99 & $(.04)$ \\
$d$ & $(.00)$ & 58.35 \\
$(\mathrm{se})$ & 1.00 & $-2,419.79$ \\
$\sigma^{2}$ & $($ fixed) & $4,878.89$ \\
LL & 16.00 & \\
BIC & $-1,532.28$ & \\
\hline
\end{tabular}

\section{Discussion}

Higher average attendance as well as a gradual increase in the daily rates was found in the period after the transition from a large to small high school arrangement. The upward jump at the beginning of the 2010-11 school year is initially followed by a relapse, but more lasting increases are observed later in the series. The findings indicate that overall, the underlying dynamics of attendance rates were favorably impacted by the small high schools implementation in the sense that they were more stable after than before the transition to the small high school format. Specifically, the analyses reveal meta-stability (edge of chaos) after the change, and instability (Brownian motion) before. These processes would have remained hidden if conventional statistics were used to describe the impact of the transition to a small high school, making the case for a thorough analysis of temporal patterns, and the engagement of educational researchers in the use of the requisite methodology.

These analyses show that attendance patterns at the Circle Academy are highly unstable prior to the intervention but that they stabilize in the post-intervention period. The long-range dependencies found in the post-intervention data suggest meta-stability, a state also referred to in the literature as the edge of chaos (Waldrop, 1992), which is to say that there is ongoing potential for transformation (for better or worse) in these attendance patterns. The results 
presented here do not address the underlying causal mechanisms to these patterns. The existing literature on small high school creation suggests that small high schools provide greater opportunity for personalized attention between students and school personnel opportunities, facilitating the engagement of students (as well as parents and communities) into the schooling process (Foley, Klinge, \& Reisner, 2008). The stabilizing impact of small high schools on daily attendance reported here may be an outcome of such a process. More research is needed to confirm this impression.

These results suggest two ways in which there is a beneficial effect of small high school creation on attendance rates in schools such as the one considered here. First, attendance rates improve, although this effect is not immediately discernible, and secondly, they stabilize, resulting in higher predictability of attendance behavior for school administrators and others who take an interest in attendance policy questions. The analyses presented here also illustrate the value of using quantitative single case designs to get a deeper understanding of the effect of our policy initiatives on educational mediators and outcome variables. The question is of particular interest in the case of transfer schools, which serve particular populations under particular conditions, for whom attending school may require students to deal with many countervailing influences.

\section{References}

Bak, P. (1996). How nature works: The science of self-organized criticality. New York: Springer.

Beran, J. (1994). Statistics for long-memory processes. Boca Raton, FL: Chapman \& Hall/CRC.

Cryer, J., \& Chan, C. (2008). Time series analysis with applications in R. New York: Springer.

Foley, E. M., Klinge, A., \& Reisner, E. R. (2008). Evaluation of New Century High Schools: Profile of an initiative to create and sustain small high schools. Washington, DC: Policy Studies Associates.

Jensen, H. J. (1998). Self-organized criticality: Emergent complex behavior in physical and biological systems. Cambridge: Cambridge University Press.

Koopmans, M. (2015a). A dynamical view of high school attendance: An assessment of short-term and long-term dependencies in five urban schools. Nonlinear Dynamics, Psychology and Life Sciences, 19(1), 65-80.

Koopmans, M. (2015b). When time makes a difference: Addressing ergodicity and complexity in education. Complicity: An International Journal of Complexity and Education, 12(2), 5-25.

Koopmans, M. (2016). Investigating the long memory process in daily high school attendance data. In M. Koopmans, \& D. Stamovlasis (Eds.) Complex dynamical systems in education: Concepts, methods and applications (pp. 299-321). New York: Springer.

Koopmans, M. (2017a). Nonlinear processes in time-ordered observations: Self-organized criticality in daily high school attendance. Complicity: An International Journal for Complexity and Education, 14(2), 78-87.

Koopmans, M. (2017b). Estimating perturbation and meta-stability in the daily attendance rates of six small high schools. Fluctuation and Noise Letters, 16(3). doi: 10.1142/S0219477517500213.

Koopmans, M. (2018). On the pervasiveness of long range memory processes in daily high school attendance rates. Nonlinear Dynamics, Psychology and Life Sciences, 22(2), 243-262.

Complicity: An International Journal of Complexity and Education

Volume 15 (2018), Number 1・pp. 19-30 
Mandelbrot, B. B. (1997). Fractals and scaling in finance: Discontinuity, concentration, risk. New York: Springer.

New York City Department of Education http://schools.nyc.gov/schoolsearch/Maps.aspx. Retrieved September 14.

Peña, D. (2001). Outliers, influential observations, and missing data. In D. Peña, G. C. Tiao, \& R. S. Tsay (Eds.) A course in time series analysis (pp. 136-170). New York: Wiley \& Sons, Inc.

Said, S. E., \& Dickey, D. A. (1984). Testing for Unit Roots in Autoregressive-Moving Average Models of Unknown Order. Biometrika 71(3), 599-607.

Sowell, F. (1992). Modeling long-run behavior with the fractional ARFIMA model. Journal of Monetary Economics, 29, 277-302.

Stadnitski, T. (2012b). Measuring fractality. Frontiers in Physiology, 3, 1-13.

Wagenmakers, E. J, Farrell, S., \& Ratcliff, R. (2004). Estimation and interpretation of $1 / \mathrm{f}^{\mathrm{f}}$ noise in human cognition. Psychonomic Bulletin \& Review, 11(4), 579-615.

\section{About the Author}

Matthijs Koopmans is a Professor in the Department of Educational Leadership at the School of Education, Mercy College. His scholarly interests include the applicability of nonlinear dynamical systems to educational organizations and school reform, cause and effect relationships and long memory processes in time series data. Correspondence: mkoopmans@mercy.edu 